\title{
cDNA analyses of CAPN3 enhance mutation detection and reveal a low prevalence of LGMD2A patients in Denmark
}

\author{
Morten Duno ${ }^{* 1}$, Marie-Louise Sveen ${ }^{2}$, Marianne Schwartz $^{1}$ and John Vissing ${ }^{2}$ \\ ${ }^{1}$ Department of Clinical Genetics, University of Copenhagen, Rigshospitalet, Copenhagen, Denmark; ${ }^{2}$ Department of \\ Neurology, Neuromuscular Research Unit, University of Copenhagen Rigshospitalet, Copenhagen, Denmark
}

Calpainopathy or limb-girdle muscular dystrophy type 2A (LGMD2A) is generally recognized as the most prevalent form of recessive LGMD and is caused by mutations in the CAPN3 gene. Out of a cohort of 119 patients fulfilling clinical criteria for LGMD2, referred to our neuromuscular clinic, 46 were suspected to have LGMD2A, based on western blot results. Four of these patients were shown to have LGMD2I upon molecular analysis, whereas 16 of the remaining 42 patients harbored mutations in CAPN3 by both direct genomic sequencing and CDNA analyses. In 10 patients, we identified both mutant alleles. In three other, only one heterozygous mutation could be identified on the genomic level; however, CAPN3 cDNA analyses demonstrated homozygosity for the mutant allele, indicating the presence of an unidentified allele that somehow compromise correct CAPN3 RNA processing. In the three remaining patients, only a single heterozygous mutation could be identified both at the genomic level and on full-length CAPN3 cDNA. All three patients exhibited a highly abnormal western blot for calpain-3 and clinical characteristics of LGMD2A. Only three of the genetically confirmed LGMD2A patients were of Danish origin, indicating a five- to sixfold lower prevalence in Denmark compared to other European countries. A total of 16 different CAPN3 mutations were identified, of which 5 were novel. The present study demonstrates the value of CDNA analysis for CAPN3 in LGMD2A patients and indicates that calpainopathy is an uncommon cause of LGMD in the Denmark.

European Journal of Human Genetics (2008) 16, 935-940; doi:10.1038/ejhg.2008.47; published online 12 March 2008

Keywords: limb-girdle muscular dystrophy; CAPN3; calpain-3; cDNA analysis; mutation screening

Introduction

Recessively inherited limb-girdle muscular dystrophy (LGMD2) comprises a heterogeneous group of patients that share the following features: (1) proximal limb weakness, (2) elevated plasma creatine kinase levels, and

${ }^{*}$ Correspondence: $\operatorname{Dr}$ M Duno, Department of Clinical Genetics, University of Copenhagen, Rigshospitalet, Blegdamsvej 9, Copenhagen DK-2100, Denmark.

Tel: + 453545 4066; Fax: + 453545 4072;

E-mail: mdunoe@rh.dk

Received 12 July 2007; revised 9 January 2008; accepted 7 February 2008; published online 12 March 2008
(3) dystrophic changes on muscle biopsy. These common features together define the diagnostic criteria for LGMD2. ${ }^{1}$ Among the LGMD2s, LGMD type 2A (LGMD2A, MIN no. 253600) is the most common and has been reported to account for at least $30 \%$ of LGMDs. ${ }^{2,3}$ The prevalence has been estimated to be around 1:100000 although with regional differences. ${ }^{3,4}$ Age of onset for LGMD2A ranges between 2 and 40 years of age, but usually manifests itself in the second or third decade of life with atrophy and weakness of pelvic and shoulder muscles. Facial and ocular muscles are spared, intellectual function is normal, and, in contrast to many other LGMDs, there seems to be no significant cardiac involvement. ${ }^{5,6}$ 
LGMD2A is caused by mutations in the CAPN3 gene, ${ }^{7}$ which encodes calpain-3; a skeletal-muscle-specific member of the calpain superfamily. ${ }^{8,9}$ Western blot for calpain-3 is usually performed as a primary diagnostic test. However, mutation screening of the CAPN3 gene is the most reliable way to verify the diagnosis, as up to $20 \%$ of molecularly confirmed LGMD2A patients show normal western blot for calpain-3. ${ }^{6,10,11}$ Normal calpain-3 possess an autolytic activity which can be used to identify LGMD2A patients with normal western blot for calpain-3. ${ }^{12,13}$ On the other hand, reduced level or absence of calpain-3 can be secondary to other genetic defects. ${ }^{6}$ The nature of this reduction is largely unknown.

The CAPN3 gene is large, consisting of 24 coding exons with an open reading frame of 2466 nucleotides, making mutation screening quite laborious and expensive. Presently, > 300 CAPN3 variants have been associated with LGMD2A. ${ }^{14,15}$ The mutations are located throughout the coding sequence, and are for most part private, although a minor subset of mutations are frequent in some populations. ${ }^{4,6,16,17}$ There is no obvious phenotype-genotype correlation, and patients with identical mutations can display quite discordant phenotypes, and vary in onset of disease. ${ }^{6,10,18}$ Moreover, African-Brazilian LGMD2A patients seem to be more severely affected than Caucasians. ${ }^{19}$ The basis for this variability remains enigmatic, but has been explained by epigenetic modulating factors ${ }^{10}$ or a second modifying allele. ${ }^{2}$

Reports from several European countries on mutation identification in CAPN3 substantiate that LGMD2A accounts for at large fraction of LGMD2s in Caucasians. ${ }^{4,20-23}$

We report here the molecular analyses of 46 patients suspected of LGMD2A, and show that the disease has an unexpected low prevalence in Denmark. In total 31 patients were analyzed for the CAPN3 cDNA sequence. Among these, the LGMD2A diagnosis was confirmed genetically in 13 patients, although some of the causative mutations remained undetected. In three other patients only one mutation could be identified on both genomic and cDNA. Thus, subset of LGMD2A patients appeared only to be heterozygous for one single CAPN3 mutation.

\section{Methods and materials \\ Subjects}

All patients were referred to our neuromuscular research unit from all over Denmark for clinical evaluation. Three were referred from clinics in Sweden. Investigations were performed upon receipt of informed consent. Initially, 119 fulfilled the diagnostic criteria for LGMD2, and of these, 59 were assigned a specific LGMD2 diagnosis other than LGMD2A, or a diagnosis of Becker muscular dystrophy (14 patients), based on molecular genetic or western blot investigations. $^{24}$ The remaining 46 patients were considered candidates for LGMD2A based on clinical and/or western blot findings. Of the 119 patients, 105 were of ethnic Danes and three were Swedes. The remaining 11 patients, who originated from different parts of the world, all belonged to the group of 46 patients who were potential candidates for LGMD2A.

\section{Western blot}

A single muscle biopsy, obtained from the vastus lateralis or tibialis anterior muscles, was snap-frozen in liquid nitrogen-cooled isopentane, stored at $-80^{\circ} \mathrm{C}$, and subsequently processed according to standard laboratory techniques. A section of each sample was tested for the presence of dystrophin, merosin, $\alpha$-sarcoglycan, $\beta$-dystroglycan, telethonin, dysferlin and calpain-3 (94, 60 and $30 \mathrm{kDa}$ fragments), using antibodies as previously described. ${ }^{24}$ The remaining of the muscle biopsy was stored for subsequent genetic analysis.

\section{Genetic analyses}

DNA was isolated from an EDTA blood sample by standard methods. All 24 coding exons and exon-intron boundaries (minimum 35 bp intronic sequence) were amplified in 22 PCR reactions (see Supplementary Table 1 for primer sequences and conditions), and either analyzed by denaturing high-pressure liquid chromatography (dHPLC) using a Wave 2100 system (Transgenomics, Elancourt, France) and/or purified on a JetQuick spin column (Genomed, St Louis, USA), directly sequenced on the forward strand using BigDyeTerminator V1.1 and subsequently resolved on an ABI3100 (Applied Biosystems, Foster City, USA). NM_000070 was used as the reference sequence for CAPN3.

Total muscle RNA was extracted from a needle biopsy using TRIzol (Invitrogen, Paisley, UK), and cDNA was generated using Superscript $\mathrm{II}^{\mathrm{TM}}$ (Invitrogen) in combination with random hexamer primers, both according to the manufacturer's description. The entire CAPN3 cDNA sequence was either amplified as one single fragment or as overlapping fragments (see Supplementary Table 1 for primer sequences and conditions) using the Expand Long Template PCR system (Roche, Basel, Switzerland).

Primers and conditions for testing mutations in FKRP have been described elsewhere. ${ }^{25}$

\section{Results}

Out of a cohort of 119 LGMD2 patients referred to our neuromuscular clinic, 46 were initially considered candidates for a LGMD2A diagnosis, based on their phenotypic appearance and/or western blot analysis. In total, 105 of the 119 patients were of Danish origin, 3 were from Sweden and the remaining 11 patients were of other ethnic background. The latter 11 all belonged to the group of 46 patients considered as potential candidates for LGMD2A. As part of the diagnostic workup, all 46 patients were tested 
for the common FKRP mutation (c.826A $>\mathrm{G}$ ), which was found in a homozygous state in four of the patients. ${ }^{24}$ These four LGMD2I patients all had abnormal western blots for calpain-3 and were ethnic Danes.

The remaining 42 patients were screened for mutations in the CAPN3 gene; 18 were initially screened by dHPLC, which confirmed the diagnosis in 4 patients. In the remaining 14 patients, the entire coding and intron flanking sequences of CAPN3 were sequenced. The other 24 patients were investigated by RT-PCR and CAPN3 cDNA sequencing. Seven of the patients, in whom no mutations were found by dHPLC/exon sequencing, were also sequenced for their CAPN3 cDNA. Mutations initially identified on the cDNA level were all confirmed by genomic sequencing.

In total, 16 different mutations were identified of which 5 were previously unknown (Table 1). The c.1117T $>$ C mutation was absent from 200 normal control chromosomes (data not shown). In 10 patients, both mutant alleles were identified, of which 3 initially were missed by dHPLC due to homozygosity. Three other patients, who appeared to carry only one mutation on genomic DNA, were shown to be homozygous on cDNA. Subsequently, the $3^{\prime}$ - and $5^{\prime}$ UTR region of these patients (nos. 4, 11 and 14) were sequenced (genomic DNA). No mutations were found. In the three remaining patients, only a single mutation was found by demonstration of heterozygosity on both genomic and cDNA (Figure 1).

The c.550delA mutation, which is relatively common in the southeastern European population, ${ }^{3,21-23}$ was not detected.

In total, 15 of the 16 mutation-positive patients had a decrease in the $94 \mathrm{kDa}$ fragment of calpain-3 on western blot (Table 1), whereas 20 of the 26 CAPN3 mutationnegative patients displayed normal western blot for calpain-3 (data not shown).

In total, 10 of the 16 mutation-positive LGMD2A patients had an ethnic background other than Danish

Table 1 The two horizontal rows in each patient referrers to the two alleles

\begin{tabular}{|c|c|c|c|c|c|c|c|}
\hline No & Western blot & Ethnicity & Genomic & $m R N A(c D N A)$ & Exon & Protein $^{\mathrm{a}}$ (predicted) & \\
\hline 1 & Absent & Somalia & $\begin{array}{l}\text { c. } 1117 T>C \\
\text { c. } 1117 T>C\end{array}$ & $\begin{array}{l}\text { r. } 1117 u>c^{b} \\
r .1117 u>c^{b}\end{array}$ & 9 & (Trp373Arg) & New \\
\hline 2 & Absent & Peru & $\begin{array}{l}\text { c.853G }>\mathrm{T} \\
\text { c. } 853 \mathrm{G}>\mathrm{T}\end{array}$ & $\begin{array}{l}r .853 \mathrm{~g}>\mathrm{t}^{\mathrm{b}} \\
\mathrm{r} .853 \mathrm{~g}>\mathrm{t}^{\mathrm{b}}\end{array}$ & 6 & (Glu285X) & New \\
\hline 3 & Highly reduced & Denmark & $\begin{array}{l}\text { c. } 257 \mathrm{C}>\mathrm{T} \\
\text { c. } 1505 \mathrm{~T}>\mathrm{G}\end{array}$ & ND & $\begin{array}{r}1 \\
11\end{array}$ & $\begin{array}{l}\text { Ser86Phe } \\
\text { lle502Ser }\end{array}$ & $\begin{array}{l}\text { Known }{ }^{26} \\
\text { New }\end{array}$ \\
\hline 4 & Absent & Denmark & $\begin{array}{l}\text { C. } 1336 \mathrm{G}>\mathrm{A} \\
\text { Not found }\end{array}$ & $\begin{array}{l}\text { r. } 1336 \mathrm{~g}>\mathrm{a} \\
\text { r.0 }\end{array}$ & 10 & (Gly446Ser) & Known ${ }^{15}$ \\
\hline 5 & Highly reduced & The Netherlands & $\begin{array}{l}\text { c. } 865 C>T \\
\text { c. } 1981 \text { delA }\end{array}$ & ND & $\begin{array}{r}6 \\
17\end{array}$ & Arg289Trp & $\begin{array}{l}\text { Known } \\
\text { Known }\end{array}$ \\
\hline 6 & Highly reduced & Denmark & $\begin{array}{l}\text { C. } 1715 G>C \\
\text { Not found }\end{array}$ & $\begin{array}{l}\text { r. } 1715 g>c \\
\text { Not found }\end{array}$ & 13 & $\begin{array}{l}\text { Arg572Phe } \\
?\end{array}$ & Known ${ }^{3}$ \\
\hline 7 & Absent & Turkey & $\begin{array}{l}\text { C. } 1309 C>G \\
\text { c. } 1309 C>G\end{array}$ & ND. & 10 & (Arg437Gly) & New \\
\hline 8 & Absent & Israel & $\begin{array}{l}\text { c. } 1800+2 T>C \\
\text { c. } 1800+2 T>C\end{array}$ & ND. & IVS15 & - & Known $^{15}$ \\
\hline 9 & Absent & Romania & $\begin{array}{l}\text { c. } 380-13 T>A \\
\text { c. } 380-13 T>A\end{array}$ & $\begin{array}{l}\text { r.380_498del1199,c } \\
\text { r.380_498del119 } 19^{\mathrm{b}, \mathrm{c}}\end{array}$ & IVS2 & (p.126fs) & New \\
\hline 10 & Highly reduced & Denmark & $\begin{array}{l}\text { c.643_663del } 21 \\
\text { Not found }\end{array}$ & $\begin{array}{l}\text { r.643_663del21 } \\
\text { Not found }\end{array}$ & 5 & $\begin{array}{l}\text { p.215_221del } \\
?\end{array}$ & Known ${ }^{26}$ \\
\hline 11 & Highly reduced & Denmark & $\begin{array}{l}\text { c. } 2393 C>A \\
(c .1801-51 G>A)^{d}\end{array}$ & $\begin{array}{l}\text { r. } 2393 c>a \\
\text { r. } 0\end{array}$ & 23 & Ala798Glu & Known $^{15}$ \\
\hline 12 & Normal & Palestine & $\begin{array}{l}\text { c. } 1381 C>T \\
\text { c. } 1381 C>T\end{array}$ & $\begin{array}{l}\text { r. } 1381 c>u^{c} \\
\text { r. } 1381 c>u^{c}\end{array}$ & 11 & Arg461Cys & Known ${ }^{35}$ \\
\hline 13 & Highly reduced & Denmark & $\begin{array}{l}\text { c.643_663del21 } \\
\text { Not found }\end{array}$ & $\begin{array}{l}\text { r.643_663del } 21^{c} \\
\text { Not found }\end{array}$ & 5 & $\begin{array}{l}\text { p.215_221del } \\
?\end{array}$ & Known ${ }^{26}$ \\
\hline 14 & Absent & Sweden & $\begin{array}{l}\text { c.2362_2362delAGinsTCATCT } \\
\text { Not found }\end{array}$ & $\begin{array}{l}\text { r.2362_2363delAGinsUCAUCU } \\
\text { r.0 }\end{array}$ & 22 & $(p .788 f s)$ & Known ${ }^{26}$ \\
\hline 15 & Absent & Russia & $\begin{array}{l}\text { c. } 1699 \mathrm{G}>\mathrm{T} \\
\text { c. } 1699 \mathrm{G}>\mathrm{T}\end{array}$ & $\begin{array}{l}\text { r. } 1699 c>u^{c} \\
\text { r. } 1699 c>u^{c}\end{array}$ & 13 & (Gly567Trp) & Known ${ }^{26}$ \\
\hline 16 & Absent & Sweden & $\begin{array}{l}\text { c.2362_2363delAGinsTCATCT } \\
\text { c.2362_2363delAGinsTCATCT }\end{array}$ & $\begin{array}{l}\text { r.2362_2363delAGinsUCAUCU }{ }^{c} \\
\text { r.2362_2363delAGinsUCAUCU }\end{array}$ & 22 & (p.788fs) & Known ${ }^{26}$ \\
\hline
\end{tabular}

Abbreviation: ND, not determined.

${ }^{a}$ Only patient no. 12 had a normal western blot for calpain-3, all other mutations ultimately lead to a quantitatively defect of functional calpain-3. ${ }^{\mathrm{b}}$ Full-length cDNA could not be generated.

${ }^{c}$ Mutation was initially identified on CDNA and subsequently confirmed on genomic DNA.

dPreviously identified as a polymorphism.

The notion ' $r .0$ ' in the CDNA column indicate that no CDNA (RNA) was detected from the corresponding allele, eg the patient was hemizygous for a mutation on the cDNA sequence but heterozygous on genomic DNA. The term 'not found' denotes a heterozygous state of the other mutation. 


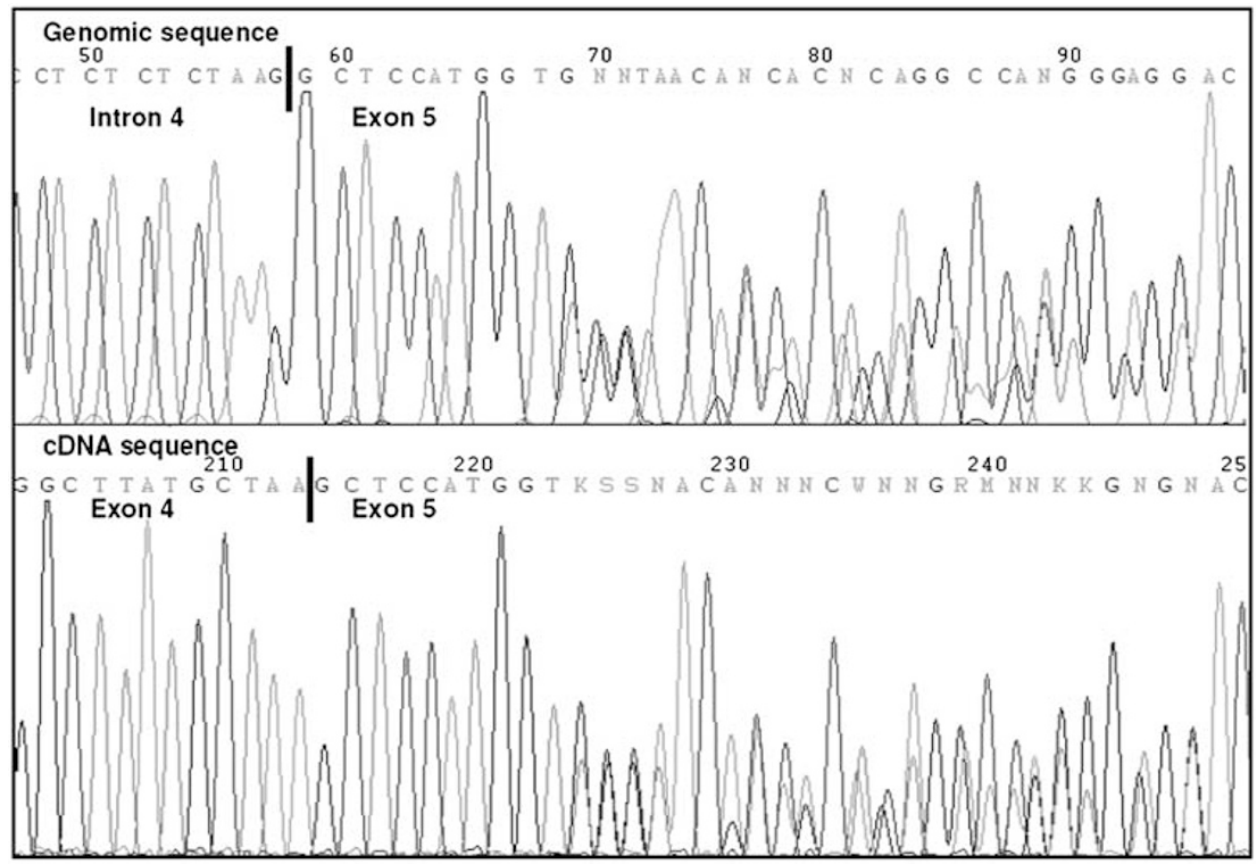

Figure 1 Electropherogram of the sequence of patient no. 13 heterozygous for c.643_663del21. Upper panel shows the genomic sequence of exon 5, whereas the lower panel depicts the cDNA sequence covering the c.643_663del21 mutation. Note that the electropherogram from the genomic and CDNA sequences are highly similar with respect to exon 5 .

(Table 1). In the remaining 103 patients with a LGMD2 phenotype, only 1 patient had a non-Danish ethnic background, indicating that the prevalence of LGMD2A in ethnic Danes is much lower than reported elsewhere in the world.

\section{Discussion}

Apart from expanding the mutation spectrum leading to LGMD2A with five new mutations, two major messages can be drawn from the present study: (1) The prevalence of LGMD2A is unexpectedly low in the ethnic Danish population, (2) cDNA analysis of CAPN3 in LGMD2A patients allows comprehensive mutation detection and can uncover the presence of mutations that escapes detection by standard genomic analysis.

Calpainopathy is recognized as the most prevalent form of recessive LGMDs and generally accounts for $>30 \%$ of LGMD2 in Caucasians. ${ }^{2,3}$ Out of a cohort of 119 LGMD2 patients (105 ethnic Danes), we found 16 carrying mutations in CAPN3. Surprisingly, only 6 were of a ethnic Danish origin, whereas the remaining 10 originated from different parts of the world, indicating that LGMD2A has a five- to sixfold lower prevalence in Denmark compared to other European countries. $4,16,21,22,26$ The reason for this remains unknown, but LGMD2I is very prevalent in Denmark, and accounts for approximately $40 \%$ of all recessive $\mathrm{LGMD}$, which is also at variance with other European countries. ${ }^{24}$ Among the 46 primary candidate patients, four were found to be homozygous for the common FKRP mutation (c.826A $>\mathrm{G})$ resulting in LGMD2I. A similar proportion of LGMD2I patients among LGMD2A referrals has been recently found in a large European cohort. ${ }^{6}$

Numerous reports have described protocols for mutation screening for the CAPN3 gene by dHPLC, ${ }^{3,10,16,22,23}$ and we initially adapted such an approach, but the failure to detect mutations in obvious candidate patients and suspicion of consanguinity, promoted us to shift to direct sequencing. Indeed, half of the molecularly confirmed LGMD2A patients turned out to be homozygous for a mutation on genomic DNA. Three of these mutations were initially missed by the dHPLC screening but could potentially have been detected by mixing DNA from the patient and a normal control (or other patent DNA) prior to dHPLC. Direct genomic sequencing is considered the gold standard for mutation detection, but as the CAPN3 gene is large, and a muscle biopsy usually is available, we changed to sequence the CAPN3 cDNA as the primary genetic examination. cDNA analysis has previously aided in the identification of CAPN3 mutations ${ }^{23,27}$ and is becoming a more widespread screening approach. ${ }^{28,29}$ To our knowledge, the majority of diagnostic laboratories perform a muscle biopsy as part of the diagnostic workup of LGMD2 patients. It should therefore be straightforward to use the biopsy for genetic analysis. cDNA analysis, however, cannot substitute genomic analysis, and identified mutations must be confirmed on genomic DNA. 
The combined genomic and cDNA sequencing led to the identification of 16 different mutations. Two of the five novel mutations (c.1309C $>\mathrm{G}$ and c. $1505 \mathrm{~T}>\mathrm{G}$ ) change the amino-acids Arg437 and Ile502, respectively, which have been found to be mutated in other LGMD2A patients ${ }^{20}$ indicating pathogenicity of the mutation, whereas two other mutations (c.853G $>\mathrm{T}$ and c.380-13T $>\mathrm{A}$ ) led to premature transcript termination due to creation of a stop codon and out of frame aberrant splicing of exon 3, respectively. How the c.380-13T $>$ A mutation interferes with the splicing machinery is unknown, but the change from a pyrimidine to a purine suggest an obstruction of the pyrimidine track. In silico analysis indicate that c.380$13 \mathrm{~T}>\mathrm{A}$ weakens the $5^{\prime}$ acceptor site of IVS2, ${ }^{30}$ supporting this suggestion. The c.1117T $>\mathrm{C}$ mutation, found in patient no. 1, leading to the amino-acid change Trp373Arg, potentially interferes with correct splicing. The c. $1117 \mathrm{~T}>\mathrm{C}$ is located next to the splice site of exon 9 , and according to the exonic splicing enhancers finder, ${ }^{31}$ the mutation changes the site preferences for various Ser-Arg-rich proteins. This in silico prediction is supported by the fact that full-length CAPN3 cDNA could not be generated, and the total absence of calpain-3 on western blot in this patient. Patient no. 1 had an affected sib that subsequently was shown to be homozygous for c.1117T- $>$ C. Thus, the five identified novel variations are all highly likely to be pathogenic.

The single c.1336G $>$ A mutation identified in patient no. 4 has not previously been described in the literature, but is listed in the Leiden database. ${ }^{15}$ The mutation changes the highly conserved hydrophobic amino-acid glycine at position 446 to a hydrophilic serine.

In 10 patients, we identified both mutant alleles. In another three, only one mutation could be identified on the genomic level (nos. 4, 11 and 14); however, CAPN3 cDNA analyses demonstrated that these patients only expressed the mutant allele, indicating that all three patients harbor an unidentified allele interfering with correct CAPN3 RNA processing. The CAPN3 transcript is targeted by the nonsense-mediated mRNA decay pathway, and a recent study has demonstrated that in LGMD2A patients, compound heterozygous for two frame-shift mutations, only trace amounts of the corresponding transcripts can be identified. ${ }^{32}$ Thus, cDNA sequencing of patients compound heterozygous for a missense, and a frame-shift/nonsense mutation will occasionally only reveal the missense mutation as the transcript carrying the premature stop-codon, depending on its location, ${ }^{33}$ will be quickly degraded. However, hemizygosity for a mutation on the cDNA level is sufficient to confirm the diagnosis. Thus, cDNA sequencing can indirectly disclose partial CAPN3 gene deletions, large genomic rearrangement, intronic or promoter mutations affecting correct CAPN3 RNA maturation/transcription, all of which are likely to be missed by traditional exon sequencing. In fact, in about $10 \%$ of LGMD2A patients, only one heterozygous mutation is identified by a genomic screening approach, ${ }^{2}$ indicating a high prevalence of deep intron mutations. In patient no. 11, we detected an intronic variation (c.1801-51G $>A$ ), which has previously been classified as a polymorphism. ${ }^{15}$ This variation is absent from the single nucleotide polymorphism database and to our knowledge not mentioned in the literature concerning CAPN3 mutation detection.

In three patients, we only identified a single variation (nos. 6, 10 and 13). Two of these were heterozygous for a $21 \mathrm{bp}$ in-frame deletion previously identified as a mutation. $^{26}$ The deletion does not appear to compromise RNA maturation, as it was clearly present on full-length cDNA (Figure 1). The third patient was heterozygous on both genomic and cDNA for the known mild mutation c. $1715 \mathrm{G}>\mathrm{C}^{3}$ All three exhibited a highly abnormal western blot for calpain-3 and clinical characteristics of LGMD2A. The parents of patients nos. 6 and 10, who carried the respective mutation, were asymptomatic, arguing against a dominant-negative effect as has been indicated for Ser86Phe. ${ }^{26}$ The lack of a normal western blot for calpain-3 is somewhat enigmatic and could point to a potential defect in translational control. To address this, both the $3^{\prime}$ - and $5^{\prime}$-UTR sequences of patient no. 6 were sequenced, but no aberration could be identified. It is quite common only to find one heterozygous mutation in LGMD2A patients, ${ }^{2}$ but to our knowledge only one patient has been described being heterozygous for a single mutation on cDNA. ${ }^{27,32}$ Interestingly, this patient carried an in-frame deletion (c.598_612del15) close to the location of the deletion found in our patients 10 and 13. A single family has been described were five male members were heterozygous for a single $3 \mathrm{bp}$ deletion in CAPN3, and all showed variable degrees of muscular dystrophy. Interestingly, all four also carried a mutation in the XK gene leading to McLeod's syndrome. ${ }^{34}$ We therefore sequenced the XK gene in patient nos. 6, 10 and 13 but did not detect any mutations. To what extent the LGMD2A pathogenesis is related to their single CAPN3 mutation alone or in combination with an unidentified mutation/locus awaits to be seen.

\section{Acknowledgements}

Excellent technical assistance was provided by Danuta Goralska-Olsen and Eva Rahtkens.

\section{References}

1 Beckmann JS, Brown RH, Muntoni F, Urtizberea A, Bonnemann C, Bushby KM: 66th/67th ENMC sponsored international workshop: the limb-girdle muscular dystrophies, 26-28 March 1999, Naarden, The Netherlands. Neuromuscul Disord 1999; 9: $436-445$.

2 Zatz M, Starling A: Calpains and disease. N Engl J Med 2005; 352: $2413-2423$ 
3 Piluso G, Politano L, Aurino $\mathrm{S}$ et al: Extensive scanning of the calpain-3 gene broadens the spectrum of LGMD2A phenotypes. $J$ Med Genet 2005; 42: 686-693.

4 Fanin M, Nascimbeni AC, Fulizio L, Angelini C: The frequency of limb girdle muscular dystrophy $2 \mathrm{~A}$ in northeastern Italy. Neuromuscul Disord 2005; 15: 218-224.

5 Pollitt C, Anderson LV, Pogue R, Davison K, Pyle A, Bushby KM: The phenotype of calpainopathy: diagnosis based on a multidisciplinary approach. Neuromuscul Disord 2001; 11: 287-296.

6 Saenz A, Leturcq F, Cobo AM et al: LGMD2A: genotype-phenotype correlations based on a large mutational survey on the calpain 3 gene. Brain 2005; 128: 732-742.

7 Richard I, Broux O, Allamand Vet al: Mutations in the proteolytic enzyme calpain 3 cause limb-girdle muscular dystrophy type $2 \mathrm{~A}$. Cell 1995; 81: 27-40.

8 Sorimachi H, Toyama-Sorimachi N, Saido TC et al: Muscle-specific calpain, p94, is degraded by autolysis immediately after translation, resulting in disappearance from muscle. J Biol Chem 1993; 268: 10593-10605.

9 Goll DE, Thompson VF, Li H, Wei W, Cong J: The calpain system. Physiol Rev 2003; 83: 731-801.

10 Fanin M, Fulizio L, Nascimbeni AC et al: Molecular diagnosis in LGMD2A: mutation analysis or protein testing? Hum Mutat 2004; 24: $52-62$.

11 Anderson LV, Davison K, Moss JA et al: Characterization of monoclonal antibodies to calpain 3 and protein expression in muscle from patients with limb-girdle muscular dystrophy type 2A. Am J Pathol 1998; 153: 1169-1179.

12 Fanin M, Nardetto L, Nascimbeni AC et al: Correlations between clinical severity, genotype and muscle pathology in limb girdle muscular dystrophy type 2A. J Med Genet 2007; 44: 609-614.

13 Fanin M, Nascimbeni AC, Fulizio L, Trevisan CP, MeznaricPetrusa M, Angelini C: Loss of calpain-3 autocatalytic activity in LGMD2A patients with normal protein expression. Am J Pathol 2003; 163: 1929-1936.

14 The Human Gene Mutation Database HGMD, http:// www.hgmd.cf.ac.uk/ac/all.php.

15 Leiden Muscular Dystrophy Database, http://www.dmd.nl/ capn3_seqvar.html.

16 Balci B, Aurino S, Haliloglu G et al: Calpain-3 mutations in Turkey. Eur J Pediatr 2006; 165: 293-298.

17 Urtasun M, Saenz A, Roudaut C et al: Limb-girdle muscular dystrophy in Guipuzcoa (Basque Country, Spain). Brain 1998; 121 (Part 9): 1735-1747.

18 Zatz M, Vainzof M, Passos-Bueno MR: Limb-girdle muscular dystrophy: one gene with different phenotypes, one phenotype with different genes. Curr Opin Neurol 2000; 13: 511-517.

19 de Paula F, Vainzof M, Passos-Bueno MR et al: Clinical variability in calpainopathy: what makes the difference? Eur J Hum Genet 2002; 10: $825-832$.
20 Richard I, Roudaut C, Saenz A et al: Calpainopathy-a survey of mutations and polymorphisms. Am J Hum Genet 1999; 64: $1524-1540$.

21 Todorova A, Kress W, Mueller C: Novel mutations in the calpain 3 gene in Germany. Clin Genet 2005; 67: 356-358.

22 Krahn M, Bernard R, Pecheux C et al: Screening of the CAPN3 gene in patients with possible LGMD2A. Clin Genet 2006; 69: $444-449$.

23 Hermanova M, Zapletalova E, Sedlackova J et al: Analysis of histopathologic and molecular pathologic findings in Czech LGMD2A patients. Muscle Nerve 2006; 33: 424-432.

24 Sveen ML, Schwartz M, Vissing J: High prevalence and phenotype-genotype correlations of limb girdle muscular dystrophy type 2I in Denmark. Ann Neurol 2006; 59: 808-815.

25 Schwartz M, Hertz JM, Sveen ML, Vissing J: LGMD2I presenting with a characteristic Duchenne or Becker muscular dystrophy phenotype. Neurology 2005; 64: 1635-1637.

26 Richard I, Brenguier L, Dincer P et al: Multiple independent molecular etiology for limb-girdle muscular dystrophy type $2 \mathrm{~A}$ patients from various geographical origins. Am J Hum Genet 1997; 60: $1128-1138$.

27 Chrobakova T, Hermanova M, Kroupova I et al: Mutations in Czech LGMD2A patients revealed by analysis of calpain3 mRNA and their phenotypic outcome. Neuromuscul Disord 2004; 14: 659-665.

28 Deburgrave N, Daoud F, Llense S et al: Protein- and mRNA-based phenotype-genotype correlations in DMD/BMD with point mutations and molecular basis for BMD with nonsense and frameshift mutations in the DMD gene. Hum Mutat 2007; 28: $183-195$.

29 Miller TE, You L, Myerburg RJ, Benke PJ, Bishopric NH: Whole blood RNA offers a rapid, comprehensive approach to genetic diagnosis of cardiovascular diseases. Genet Med 2007; 9: $23-33$.

30 Reese MG, Eeckman FH, Kulp D, Haussler D: Improved splice site detection in Genie. J Comput Biol 1997; 4: 311-323.

31 ESE finder, http://rulai.cshl.edu/cgi-bin/tools/ESE3/esefinder.cgi.

32 Stehlikova K, Zapletalova E, Sedlackova J et al: Quantitative analysis of CAPN3 transcripts in LGMD2A patients: involvement of nonsense-mediated mRNA decay. Neuromuscul Disord 2007; 17: 143-147.

33 Maquat LE: Nonsense-mediated mRNA decay: splicing, translation and mRNP dynamics. Nat Rev Mol Cell Biol 2004; 5: 89-99.

34 Starling A, Schlesinger D, Kok F, Passos-Bueno MR, Vainzof M, Zatz M: A family with McLeod syndrome and calpainopathy with clinically overlapping diseases. Neurology 2005; 65: $1832-1833$

35 Minami N, Nishino I, Kobayashi O, Ikezoe K, Goto Y, Nonaka I: Mutations of calpain 3 gene in patients with sporadic limb-girdle muscular dystrophy in Japan. J Neurol Sci 1999; 171: 31-37.

Supplementary Information accompanies the paper on European Journal of Human Genetics website (http://www.nature.com/ejhg) 